\title{
Benjamin Steiner, Colberts Afrika. Eine Wissens- und Begegnungsgeschichte in Afrika im Zeitalter Ludwigs XIV.
}

\author{
Claire Gantet
}

\section{OpenEdition}

\section{Journals}

Édition électronique

URL : http://journals.openedition.org/ifha/8749

DOI : $10.4000 /$ ifha. 8749

ISSN : 2198-8943

\section{Éditeur}

IFRA - Institut franco-allemand (sciences historiques et sociales)

\section{Référence électronique}

Claire Gantet, «Benjamin Steiner, Colberts Afrika. Eine Wissens- und Begegnungsgeschichte in Afrika im Zeitalter Ludwigs XIV. », Revue de I'IFHA [En ligne], Date de recension, mis en ligne le 02 novembre 2017, consulté le 23 septembre 2020. URL : http://journals.openedition.org/ifha/8749 ; DOI : https://doi.org/ 10.4000/ifha.8749

Ce document a été généré automatiquement le 23 septembre 2020

(CIFHA 


\title{
Benjamin Steiner, Colberts Afrika.
} Eine Wissens- und Begegnungsgeschichte in Afrika im Zeitalter Ludwigs XIV.

\author{
Claire Gantet
}

\section{RÉFÉRENCE}

Benjamin Steiner, Colberts Afrika. Eine Wissens- und Begegnungsgeschichte in Afrika im Zeitalter Ludwigs XIV., München: De Gruyter/Oldenbourg, 2014, xii-483 p., 79,95€ 
L'Afrique de Colbert: une histoire du savoir et de la rencontre en Afrique à l'époque de Louis $X I V$, tel est le projet neuf et ambitieux que Benjamin Steiner développe dans sa thèse d'habilitation. Tandis que les courtisans qui commencent à s'installer à Versailles ne connaissent rien de l'Afrique, une bureaucratie (de petits nobles et des bourgeois) est mise en place, en particulier sous l'action de Colbert, laquelle pratique une politique d'information et d'implantation en Sénégambie, à Madagascar et dans les Mascareignes. Tandis que les grands nobles s'intéressent à l'Europe, les plus petits regardent vers l'Afrique qui semble leur offrir des possibilités d'ascension sociale. Non seulement l'Afrique est pleinement présente dans la politique administrative ludovicienne, mais elle

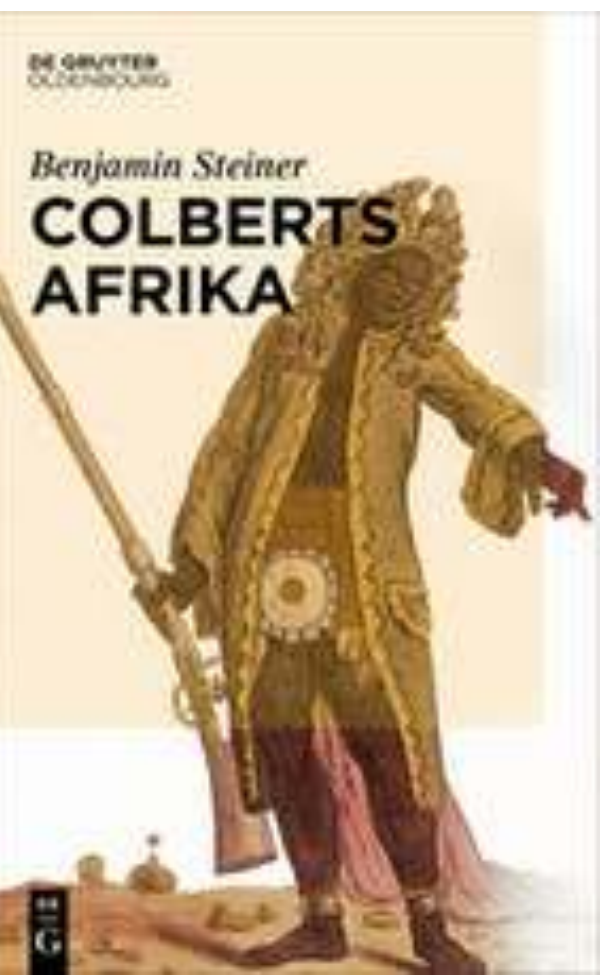
marque aussi de son empreinte le développement de l'État en France même.

L'histoire de la présence française en Afrique est mal connue : d'abord apologétique du pouvoir (jusqu'à l'ouvrage de Pierre Pluchon et au recueil dirigé entre autres par Jean Meyer et Jean Tarrade, toujours consultés), puis critique, elle reste pour l'époque moderne à l'ombre de celle de l'Amérique. Empreinte du "discours de la séparation » défini par Michel de Certeau, l'historiographie francophone, jusqu'aux ouvrages de Tzvetan Todorov, sonde le rabattement de l'étranger sur le même en une herméneutique de l'autre qui tend à essentialiser des catégories éminemment mouvantes. Plutôt que de se pencher sur l'altérité, B. Steiner propose de sonder des « rencontres ». Loin de s'enfermer dans une histoire étroitement politique, il ouvre toutefois sa perspective à l'histoire des savoirs et de l'information. Contrairement à la perspective de la «science moderne" qui repose sur le postulat plus ou moins tacitement accepté d'une supériorité de la science européenne, il pose que le savoir naît dans des contextes de rencontre de nombreux acteurs. Les compagnies de commerce tinrent lieu de plateformes d'information, que l'État tenta de monopoliser, d'utiliser et d'étayer. Une véritable "culture du mémorandum» se développa sous l'égide de Colbert. L'intérêt pour les pratiques informatives généra des missions exploratoires et un savoir scientifique.

B. Steiner développe son propos (de façon très française!) en trois parties, chacune (hormis la première) étant divisée en trois sous-parties. La première partie est une contextualisation destinée à comprendre pourquoi la France, alors un pays puissant et peuplé, n'est pas parvenue à mettre en place un Empire colonial à l'égal de l'Espagne et du Portugal. Une première réponse envisage les dysfonctionnements de l'appareil d'État engendrés par les conflits confessionnels jusque vers 1650, malgré quelques tentatives de mission. Sous le règne de Louis XIv, très marqué par les guerres, l'Afrique n'est pas absente. Bien au contraire, Gorée est prise dès 1677 et les Hollandais sont 
expulsés du Sénégal après 1678 ; entre 1688 et 1714, le commerce d'asiento est étendu à la Guinée, et le territoire de la Sénégambie est consolidé. Des premières compagnies de commerce, locales et non centrales, ont l'initiative de voyages de découverte et implantent des établissements dans des villes portuaires. Leurs intérêts allant toutefois à l'encontre de ceux de l'État, Colbert s'attache à monopoliser le commerce avec l'Amérique, l'Afrique et l'Asie, liant progressivement les pratiques informatives économiques et étatiques en une structure administrative fondée sur l'information.

Le plan d'un «système commercial global nécessitait [toutefois] une autre forme d'acquisition et de hiérarchisation du savoir» (p.124). C'est en quête de l'«État informé » que B. Steiner part dans la deuxième partie, où il sonde les tensions entre la notion d'État souverain et la pratique sociale du patronage. Le gouvernement français parvient à imposer son pouvoir malgré la distance au moyen de techniques administratives et de pratiques informatives. L'échec de la colonisation de Madagascar révèle les difficultés de telles entreprises dans un contexte de faible densité administrative et de relative autonomie des envoyés. À long terme néanmoins, les pratiques de correspondances et de mémorandums s'avèrent fructueuses. Par-delà la durée de vie courte des compagnies de commerce, le personnel reste stable, si bien qu'il peut servir les visées du gouvernement. L'alliance de l'argent et des organes de décision permet en France un développement du ministériat.

La dernière partie sonde l'efficacité du régime informationnel dans des situations de rencontre entre Français et Africains. Avant même l'implantation de l'État français, des négociants et des missionnaires s'établissent dans un Empire portugais chancelant. Grâce à leurs relations avec les chefs indigènes, ils y acquièrent un fond de connaissances sur les pays et leurs habitants. Des contacts commerciaux français sont progressivement établis en Sénégambie et en Guinée, puis l'administration royale anime des missions de découverte et d'exploitation d'autres terres, dans l'intérieur de l'Afrique, qui aboutissent à une maîtrise durable de la Sénégambie. Cette tendance est soutenue par la diffusion d'une image positive des mœurs africaines en France. Au Sénégal, des acteurs français ont à l'occasion instrumentalisé leur séjour pour se positionner comme découvreurs, entrepreneurs et intermédiaires nécessaires, monter ainsi dans la hiérarchie sociale ou s'enrichir aux dépens des visées bureaucratiques. Sur place toutefois ne domine pas un net clivage entre les arrivants et les indigènes, mais plutôt le souci de vivre en bonne intelligence.

$\mathrm{Au}$ terme de ce livre, le lecteur peut se demander pourquoi les fortes pertes démographiques des Européens n'apparaissent guère dans l'information relative à l'Afrique, et relever un certain nombre de coquilles dans les citations françaises. Il n'en reste pas moins que ce livre, étayé sur une vaste documentation archivistique, jette un nouveau regard sur la présence française en Afrique dans un long XVII siècle, tout en évitant les chausse-trapes de l'histoire coloniale et en privilégiant une approche neuve en termes d'histoire des savoirs. On reconnaitra l'influence de certains thèmes de l'ouvrage important d'Arndt Brendecke sur la fonction du savoir dans l'édification de l'Empire colonial espagnol (Imperium und Empirie. Funktionen des Wissens in der Spanischen Kolonialherrschaft, Cologne et al., 2009), signe d'une importante collégialité. Écrit dans une langue limpide et structuré en une progression très claire, cet ouvrage montre de façon tout à fait convaincante l'intérêt pour l'Afrique des organes gouvernementaux et de pans de la population française, le rôle du savoir (plus que des capitaux) dans l'implantation française outre-mer et l'influence des pratiques informatives qui y ont 
été éprouvées dans le développement de l'absolutisme. On ne peut donc qu'en souhaiter une ample réception en France - et au-delà.

INDEX

Index chronologique : Période moderne

Thèmes : Histoire des États et des pouvoirs, Histoire des sciences, Histoire des idées

\section{AUTEURS}

\section{CLAIRE GANTET}

Université Fribourg (Suisse)/Universität Freiburg (Schweiz) 